\title{
System and Method for 3-D/3-D Registration between Non-contrast-enhanced CBCT and Contrast-Enhanced CT for Abdominal Aortic Aneurysm Stenting
}

\author{
Shun Miao ${ }^{1}$, Rui Liao ${ }^{1}$, Marcus Pfister ${ }^{2}$, Li Zhang $^{1}$, and Vincent Ordy ${ }^{1}$ \\ 1 Siemens Corporation, Corporate Technology, Princeton, NJ, USA \\ 2 Siemens Healthcare, Erlangen, Germany
}

\begin{abstract}
In this paper, we present an image guidance system for abdominal aortic aneurysm stenting, which brings pre-operative 3-D computed tomography (CT) into the operating room by registering it against intra-operative non-contrast-enhanced cone-beam CT (CBCT). Registration between $\mathrm{CT}$ and $\mathrm{CBCT}$ volumes is a challenging task due to two factors: the relatively low signal-to-noise ratio of the abdominal aorta in CBCT without contrast enhancement, and the drastically different field of view between the two image modalities. The proposed automatic registration method handles the first issue through a fast quasi-global search utilizing surrogate 2-D images, and solves the second problem by relying on neighboring dominant structures of the abdominal aorta (i.e. the spine) for initial coarse alignment, and using a confined and image-processed volume of interest around the abdominal aorta for fine registration. The proposed method is validated offline using 17 clinical datasets, and achieves $1.48 \mathrm{~mm}$ target registration error and $100 \%$ success rate in $2.83 \mathrm{~s}$. The prototype system has been installed in hospitals for clinical trial and applied in around 30 clinical cases, with $100 \%$ success rate reported qualitatively.
\end{abstract}

Keywords: 3-D/3-D registration, cone-beam computed tomography, abdominal aortic aneurysm, global optimization.

\section{Introduction}

Abdominal aortic aneurysm (AAA) causes 15,000 death yearly in the U.S. 1]. As an alternative to the well-established open surgery, minimally invasive AAA stenting is a rapidly emerging technology that is especially suitable for high-risk surgical candidates. Contrast-enhanced computed tomography (CT) provides detailed anatomical assessment of the abdominal aorta and therefore is routinely performed in pre-operative planning. Overlay of pre-operative CT and intra-operative fluoroscopy is also used during the procedure, especially for complicated aneurysms, to guide the catheterization of the side branches and positioning of the stents. However, the current clinical practice is hindered by the cumbersome workflow, including (semi-)manual registration. 

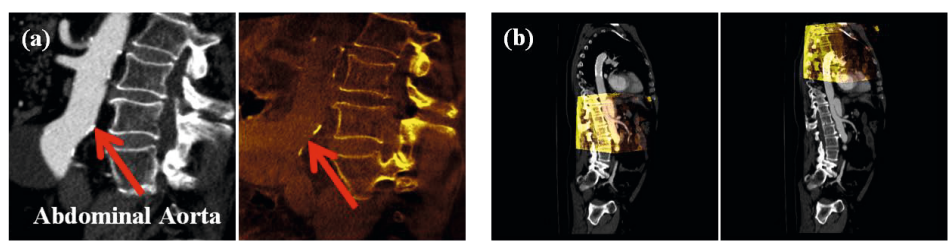

Fig. 1. (a) Sagittal slices of CT (white) and CBCT (yellow). Abdominal aorta (pointed out by red arrows) has high contrast in contrast-enhanced $\mathrm{CT}$, but is barely visible in non-contrast-enhanced CBCT. (b) Overlay of CT (white) and CBCT (yellow) before and after registration, showing the large difference in FOVs and the need for a global optimization scheme.
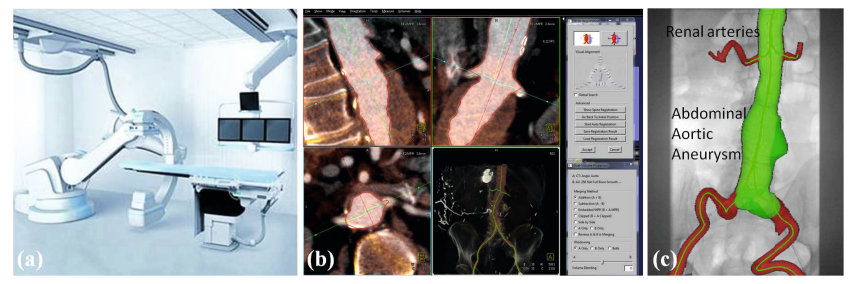

Fig. 2. (a) Angiographic C-Arm system to acquire interventional 3-D CBCT volumes in an operating room. (b) 3-D/3-D registration system for AAA stenting. (c) Overlay of the 3 -D model on a real-time $2-\mathrm{D}$ fluoroscopy.

This paper describes a $3-\mathrm{D} / 3-\mathrm{D}$ registration system that brings the preoperative contrast-enhanced $\mathrm{CT}$ into the operating room by registering it against the intra-operative non-contrast-enhanced cone-beam CT (CBCT). Because renal insufficiency is highly prevalent in patients undergoing AAA stenting, extensive use of iodine contrast may cause renal failure and needs to be avoided. Therefore, we use non-contrast CBCT for registration purpose, which is a challenging problem due to the relatively poor image quality of the non-contrastenhanced CBCT, where the abdominal aorta has a very low image contrast (Fig. 1a). Another challenge is that abdominal CT typically has a much larger field of view (FOV) than CBCT (Fig. 10), leading to many local optima in the registration space.

To the best of our knowledge, there is no literature reporting on automatic registration of abdominal aorta between contrast-enhanced CT and non-contrastenhanced CBCT due to the aforementioned difficulties. Normalized Mutual Information (NMI) has been widely applied for general multi-modality image registration [2]. However NMI is driven mainly by large structures with high contrast and therefore is not directly applicable to our application, where the target object to be registered is relatively small and homogeneous compared to other neighboring structures. Multi-resolution strategy has been used with local optimizers for an increased capture range in registration [3], which, however, does not work adequately when the FOVs vary in a great extent like in our case. Global 
search has been mainly limited to 2-D image registration tasks due to its high computational complexity. Some heuristic semi-global optimization schemes, e.g. simulated annealing [4] and genetic algorithm [5], are proposed for the trade off between capture range and efficiency. However, their computational cost for 3$\mathrm{D} / 3-\mathrm{D}$ registration is still prohibitively high for interventional use.

\section{System Overview}

Our 3-D/3-D registration system has been prototypically integrated into an angiographic C-arm system (Siemens Artis zee/zeego, Fig. 2a). Fig. 2b shows the prototype system with an example registration. Mesh models are used for representing the abdominal aorta segmented from CT, which is then overlaid onto intra-operative 2-D X-ray images for the purpose of real-time navigation and guidance during AAA stenting procedures (Fig. 2r).

To facilitate visual check of registration accuracy by physicians, CT and CBCT volumes are displayed and blended in both volume rendering and multiplanar reformatting (MPR). Multiple options are provided for the blending effect for an intuitive check, such as summation, subtraction, side by side, and embedded MPR of CT into CBCT volume. Cutting planes of the MPRs are automatically determined based on the abdominal aorta segmented from CT and the detected landmarks including renal artery ostia and illiac artery bifurcations, so that physicians can verify the registration result at the target area without any manual operation.

\section{Registration Method}

\subsection{Quasi-global Search}

A good pose initialization is important for intensity-based registration methods, especially for our application where the FOVs of CT and CBCT volumes are dramatically different. However, a complete global search in 3-D space is not computationally practical. To achieve a reliable and efficient initialization, we follow the concept of using 2-D anatomy targeted projections to surrogate the original volume 6]. A similarity measure is then defined on the lower-dimensional (2-D) surrogate images, instead of the 3-D volume, which significantly reduces the computation cost of similarity evaluation, thereby making quasi-global search in the registration space computationally feasible.

To compute the surrogate image, the Maximum Intensity Projections (MIPs) are computed along three directions $(x, y, z)$, as shown in Fig. 3. For example, given the CT volume $I_{C T}$, the surrogate image of CT along $x$ direction is defined as:

$$
M C T_{x}=\max _{x} I_{C T}(x, y, z)
$$

Surrogate images along $x, y, z$ directions are denoted as $M C T_{x}, M C T_{y}, M C T_{z}$ for $\mathrm{CT}$ and $M C B_{x}, M C B_{y}, M C B_{z}$ for CBCT, respectively. The similarity between CT and CBCT volumes for the purpose of pose initialization is then defined as the summation of the NMIs of all three pairs of surrogate images: 

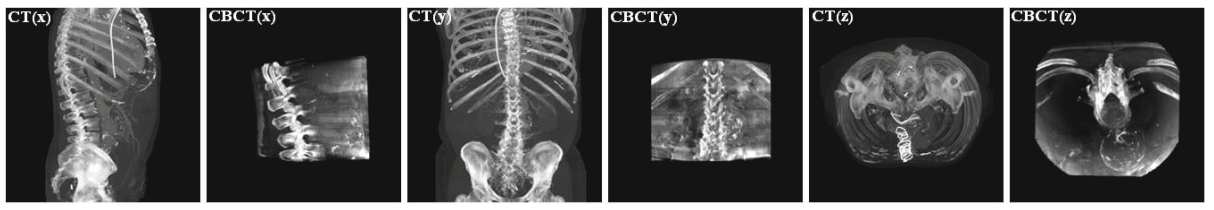

Fig. 3. Surrogate images for CT and CBCT
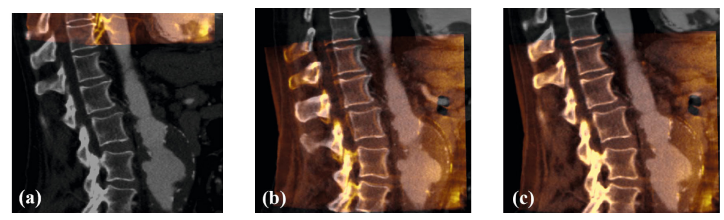

Fig. 4. (a) Initial position before registration. (b) Registration using the whole volume. (c) Registration using the spine segmentation.

$$
S\left(I_{C T}, I_{C B}\right)=\sum_{i=x, y, z} N M I\left(M C T_{i}, M C B_{i}\right)
$$

For AAA intervention, typically both CT and CBCT volumes are acquired with the patient lying in the supine position. Therefore the two volumes have similar initial orientations and the main objective of pose initialization is to estimate 3-D translation. If the transformation $T$ is translation-only, the similarity can be computed as:

$$
S\left(T\left(I_{C T}\right), I_{C B}\right)=\sum_{i=x, y, z} N M I\left(T_{i}^{2 D}\left(M C T_{i}\right), M C B_{i}\right)
$$

where $T_{i}^{2 D}, i=x, y, z$ are the corresponding 2-D translations of the three surrogate images using the $3-\mathrm{D}$ translation parameters from $T$. Note that 2-D translation of the surrogate images as a result of $3-\mathrm{D}$ translation of the original volume can be computed efficiently without re-computation of the MIPs.

Several hundred positions in the registration space are sampled, and translation only local optimization in multi-resolution pyramid is performed starting from each selected position based on the similarity measure defined in Eqn. 3 . In addition, the spatial location of the structure of interest in image domain and the likely location of local optima in parameter domain are used to best choose the starting points globally in the registration space. For example, denser and more points are sampled in the head-foot direction because it is known that the largest variance between the FOVs of CT and CBCT volumes lies in this direction, and local optima happen more likely along this direction due to the repetition pattern of the spine in thoracic-abdominal CT/CBCT. Pose initialization is then obtained as the global optimal position with the highest similarity measure among all the registration results. 


\subsection{Spine Registration}

After coarse alignment of CT and CBCT volumes through pose initialization, a structure-targeted rigid-body registration is performed to align the spine, which is a dominant structure in both image modalities and as a result can be much more reliably registered in this step compared to the abdominal aorta. In addition, registration of the spine brings the abdominal aorta close to the correct position because these two structures are anatomically adjacent to each other.

To focus the registration on the spine, we first coarsely segment the spine from both $\mathrm{CT}\left(P_{C T}\right)$ and $\mathrm{CBCT}\left(P_{C B}\right)$ volumes via thresholding using the known range of Hounsfield Unit (HU) for bony structures. This simple and coarse segmentation is sufficient for registration purpose because the most dominant features of the spine, such as the bright edge of the vertebrae, can always be reliably segmented. In addition, those large organs that potentially interfere with spine registration, such as the liver, can be reliably excluded. The spine segmentation results from both $\mathrm{CT}$ and CBCT volumes are combined as:

$$
P=P_{C T} \cap P_{C B}
$$

And the NMI similarity measure is defined on the spine as

$$
N M I_{s}\left(I_{C T}, I_{C B}\right)=\frac{H\left(I_{C T}(P)\right)+H\left(I_{C B}(P)\right)}{H\left(I_{C T}(P), I_{C B}(P)\right)}
$$

where $H\left(I_{1}\right)$ and $H\left(I_{2}\right)$ are entropy of $I_{1}$ and $I_{2}$, respectively, and $H\left(I_{1}, I_{2}\right)$ is the joint entropy. By defining the NMI similarity measure on the spine only, the registration is targeted and as a result most accurate on the spine, as shown in Fig. 4. In addition, to ensure the smoothness of the joint histogram and thus reduce the number of local optima in the registration space, we estimate the joint histogram on the basis of Parzen windows made of Gaussian density function [7.

\subsection{Aorta Registration}

Registration of the abdominal aorta is difficult due to the very low signal-to-noise ratio of the vessel structures in CBCT without contrast medium. In addition, for AAA stenting, the main stent graft needs to be deployed very close to the renal artery ostia with a sufficient supporting zone but without occluding them. Registration thus needs to be highly accurate in the area around renal artery ostia, which is a relatively small object compared to other neighboring structures, such as the spine, the liver, and etc. To handle this problem, we use a confined and image-processed VOI around the abdominal aorta and the renal ostia, to eliminate irrelevant interfering structures and enforce the registration algorithm focusing on the target organ for aorta registration.

To calculate the confined VOI, we first automatically segment vessel structures from CT using a graph-cut based method [8]. This segmentation is straightforward because vessel structures are contrast-enhanced in the CT volume. The VOI in CT is then defined as a bounding box that contains the segmented renal 

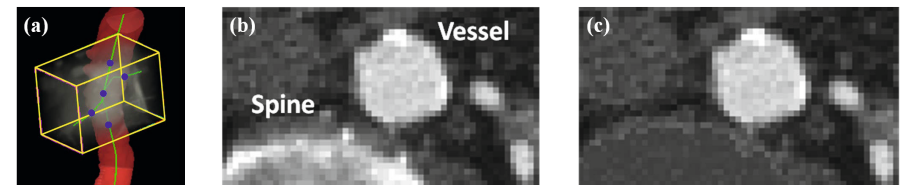

Fig. 5. (a) Abdominal aorta segmentation (red), centerline (green) and VOI automatically calculated based on the segmentation (yellow). Five target points that are used for computing TRE are shown as blue dots. (b) Spine is included in the VOI because it is very close to the abdominal aorta. (c) Spine is eliminated from the VOI by the proposed spine removal process.

arteries, the renal ostia and the upper part of the abdominal aorta, as shown in Fig. 5a. Since the abdominal aorta in CBCT is already relatively close to that in $\mathrm{CT}$ after spine registration, the VOI in CBCT is a dilated version of the VOI in CT, eliminating the need for explicit segmentation on CBCT which is a much more difficult task without contrast enhancement.

The spine can still possibly be included in the confined VOI due to its close proximity to the abdominal aorta (Fig. 5b). To eliminate the impact of the spine, image processing is applied on the CT to remove the spine. Once the spine is removed from the $\mathrm{CT}$, the spine in the $\mathrm{CBCT}$ has no matching structure and therefore has little influence during registration process. The spine mask is generated by thresholding CT intensities within the VOI by a heuristic HU value corresponding to the bone and excluding the voxels in the vessel segmentation. The intensity mean and variance of the VOI excluding the spine and the vessel are computed and denoted as $\mu$ and $\sigma$, respectively. The spine is then removed by filling those spine voxel with intensities from a Gaussian distribution $N(\mu, \sigma)$, as shown in Fig. 55.

In the confined and image-processed VOI, the abdominal aorta and the renal arteries become the only dominant structures, and therefore can be reliably registered using standard intensity-based registration methods, starting from the position obtained by spine registration. We again use NMI as the similarity measure and Hill Climbing (HC) as the optimizer. Two examples of aorta registration are shown in Fig. 6.

\section{Algorithm Implementation}

Our system is based on a highly optimized implementation: 1. Multi-resolution strategy is used in all steps. 2. Recursive Gaussian filtering is used in Parzen window joint histogram estimation for computational efficiency [9]. Furthermore, the implementation of the filtering is optimized to ensure coalesce memory access. 3 . A relatively small joint histogram $(128 \times 128 \mathrm{bins})$ is used to reduce the cost of evaluating NMI, and the upper and lower bound of the histogram are carefully chosen to ensure sufficient intensity resolution of the target object. 4. OpenMP is used to take advantage of multi-core CPU. 

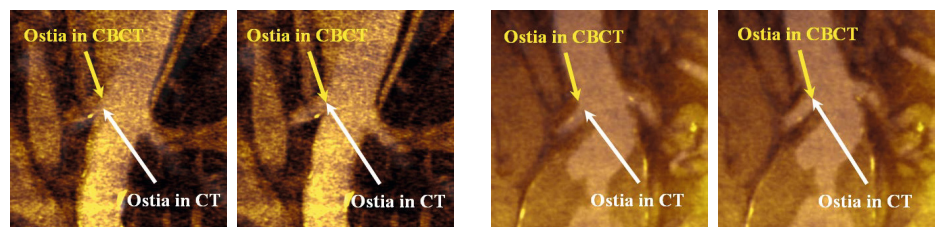

Fig. 6. Two examples of CT and CBCT overlay before (left) and after (right) aorta registration. Renal ostia (annotated by arrows) are about $7 \mathrm{~mm}$ off originally, and are perfectly aligned after aorta registration.

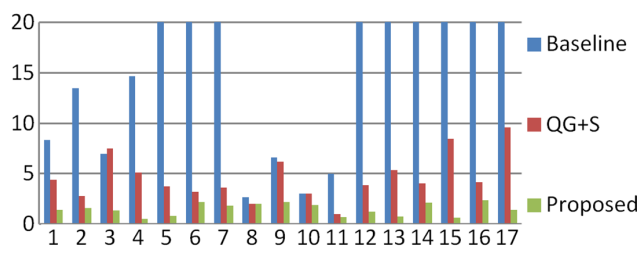

Table 1. Summary of Results

\begin{tabular}{lccc}
\hline & \multicolumn{3}{c}{$\mu$ TRE maxTRE Success } \\
& $(\mathrm{mm})$ & $(\mathrm{mm})$ & rate \\
\hline Baseline & 43.15 & 176.15 & $5.89 \%$ \\
QG+S & 4.59 & 9.56 & $23.53 \%$ \\
Proposed & 1.48 & 2.39 & $100 \%$ \\
\hline
\end{tabular}

Fig. 7. TRE for 17 datasets

\section{$5 \quad$ Experiments and Results}

We conducted experiments using 17 AAA clinical datasets to validate the proposed method. Registration accuracy is measured by 3-D target registration error (TRE), which is defined as the average 3-D Euclidean distance between the transformed landmarks and the corresponding ground truth (manually annotated and confirmed by domain experts) for 5 landmarks: 2 renal ostia and 3 points along the centerline of the abdominal aorta. We calculated the average TRE ( $\mu$ TRE) and the maximum TRE (maxTRE) for all datasets. Success rate is evaluated based on the criterion that $\mathrm{TRE}<2.5 \mathrm{~mm}$ is clinically useful (according to our collaborating physicians) and thus a successful registration.

Experimental results are summarized in Table 1 and Fig. (7) In Fig. [7, the errors by baseline registration for some datasets far exceeding the range are not fully plotted. We first evaluated the baseline intensity-based 3-D/3-D registration, which uses NMI as the similarity measure and $\mathrm{HC}$ as the optimizer. The initial position before registration is provided by overlaying the center of mass of the two volumes. This method has a small capture range, leading to a low success rate $(5.89 \%)$ and a large $\mu \mathrm{TRE}(43.15 \mathrm{~mm})$. We also evaluated the performance of applying only the first two steps (quasi-global search and spine registration, denoted as QG+S) of the proposed method. This method achieved maxTRE of $9.56 \mathrm{~mm}$, demonstrating that it successfully brings the target object close to the correct position for all the test cases. However, the $\mu \mathrm{TRE}(4.59 \mathrm{~mm})$ and the low success rate $(23.53 \%)$ indicates that the registration accuracy in the target area after these two steps is insufficient for clinical use. By performing the last step of aorta registration, the proposed method achieved $\mu \mathrm{TRE}$ of $1.48 \mathrm{~mm}$ and $100 \%$ success rate. 
Due to the high efficiency of the proposed algorithm for quasi-global search as well as our highly optimized implementation, our method achieved $2.83 \mathrm{~s}$ computation time on average (Intel Xeon E5-5620, volume size $512 \times 512 \times 781$ ), which is critical for the system to be well accepted clinically. This is about one order of magnitude faster compared to other existing methods. For example, DIRECT (Dividing Rectangles) deterministic global optimization algorithm takes $36 \mathrm{~s}$ to complete 3D3D registration using 8 CPUs [10].

Besides offline experiments, our system is currently under clinical trial and has been used during 30 clinical cases. So far we have received $100 \%$ success rate from qualitative clinical feedbacks. This system meets the clinical requirements of AAA stenting procedures in terms of accuracy, robustness and speed.

\section{Discussions and Conclusions}

In this paper, we presented an image guidance system that brings pre-operative CT into the operating room to support interventional AAA stenting. The proposed 3-D/3-D registration method is fully automatic and highly efficient, and the system (including visualization) is seamlessly integrated into AAA stenting workflow. The presented work focus on the initial alignment of CT volumes and the support on the deployment of AAA main stent graft. Our future works include: 1. 2-D/3-D registration to compensate for patient movement during the procedure. 2. local deformable registration of iliac arteries to support the deployment of the branch stent graft during AAA.

\section{References}

1. Creager, M.A., et al.: Aneurysmal disease of the aorta and its branches. In: Vascular Medicine: A Textbook of Vascular Biology and Disease, pp. 901-925 (1996)

2. Pluim, J.P.W., et al.: Mutual-information-based registration of medical images: a survey. TMI 22(8), 986-1004 (2003)

3. Thévenaz, P., et al.: Optimization of mutual information for multiresolution image registration. TMI 9(12), 2083-2099 (2000)

4. Matsopoulos, G.K., et al.: Automatic retinal image registration scheme using global optimization techniques. TITB 3(1), 47-60 (1999)

5. Rouet, J.M., et al.: Genetic algorithms for a robust 3-d mr-ct registration. TITB 4(2), 126-136 (2000)

6. Zhang, L., et al.: A knowledge-driven quasi-global registration of thoracicabdominal ct and cbct for image-guided interventions. In: SPIE (2013)

7. Wells, W.M., et al.: Multi-modal volume registration by maximization of mutual information. Medical Image Analysis 1(1), 35-51 (1996)

8. Lombaert, H., et al.: A multilevel banded graph cuts method for fast image segmentation. In: ICCV, vol. 1, pp. 259-265. IEEE (2005)

9. Gunnar, F., et al.: Improving deriche-style recursive gaussian filters. JMIV 26(3), 293-299 (2006)

10. Dru, F., et al.: An itk framework for deterministic global optimization for medical image registration. In: Medical Imaging, p. 61442. International Society for Optics and Photonics (2006) 